\title{
VERIFICATION OF NANOINDENTATION DEVICES USING ATOMIC FORCE MICROSCOPY
}

\author{
1'Anna CHARVÁTOVÁ CAMPBELL, 1,2Marek HAVLÍČEK, ${ }^{1,2}$ Petr KLAPETEK, ${ }^{3}$ Vilma BURŠíKOVÁ \\ ${ }^{1}$ Czech Metrology Institute, Brno, Czech Republic, EU, \\ acampbellova@cmi.cz \\ ${ }^{2}$ Central European Institute of Technology, Brno, Czech Republic, EU \\ ${ }^{3}$ Faculty of Science, Masaryk University, Brno, Czech Republic, EU
}

https://doi.org/10.37904/nanocon.2019.8633

\begin{abstract}
Nanoindentation has become a popular tool for the exploration of mechanical properties of materials at the nanoscale. However, calibration and traceability issues are often neglected, making the direct comparison of results between different instruments difficult. The direct calibration of the sensors is challenging and requires dedicated instruments leaving indirect calibration as the only option to ordinary users. However, indirect calibration compares only the resulting hardness or modulus of the reference sample and the measured sample. Therefore, it cannot distinguish the source of errors, such as errors in the depth and load sensors, errors in the reference sample properties, contamination of the tip or sample, wear of tip and others.

In this contribution we explore the possibility of using measurements of indents by atomic force microscopy for the verification of the depth sensor. Measurements for different nanoindentation devices are presented and compared with a focus on the uncertainties of the method.
\end{abstract}

Keywords: Nanoindentation, depth sensor, calibration, AFM

\section{INTRODUCTION}

Nanoindentation has been used for over more than two decades to study the mechanical properties of objects at the nanoscale, such as thin films, nanoparticles, composites, nanotubes and others. It is based on pushing a sharp hard tip, usually made of diamond, into the sample and measuring continuously the load applied to the tip and the depth into which it penetrates the sample. From these load and depth data as well as information about the tip shape, various material properties can be acquired: the most common being the indentation hardness and Young's modulus following the method by Oliver and Pharr [1].

In order to acquire meaningful, quantitative data which can be compared with results from other laboratories, metrological aspects such as traceability are crucial. Traceability consists of an unbroken chain of measurements, so-called calibrations, which link the given measurement including its uncertainty to other instruments up to national standards [2].

The calibration of highly complex instruments, such as the nanoindenter, is non-trivial [3-5]. The sensors are usually calibrated by the manufacturer but often this procedure is not traceable and insufficient information is available. Furthermore, the instrument's performance may change due to ageing, which requires a recalibration. Obviously, it is also necessary to check the instrument regularly, as the changes may be minor at first and can be overseen in the beginnings. Calibration of complex instruments is quite often very problematic, mainly due to bad access to sensors. Solutions to this problem may require special equipment, which may be costly or require expertise not available in the given laboratory. Therefore, these complex instruments are very often calibrated indirectly using reference samples. In this case, not the sensors themselves are calibrated but a chosen output quantity, e.g. modulus or height. In the case of a nanoindenter, 
the height sensor, load sensor, frame compliance and tip shape must be calibrated. Calibration is most commonly performed using reference samples with specified hardness or elastic modulus but this does not allow to study the performance of the height or load sensors themselves. In fact, once the system is calibrated, reasonable results for elastic modulus can be obtained even though the intermediate results of depth and load are false. This is an advantage for the measurement of elastic modulus, but a problem for the study of depth itself. For the direct calibration of depth sensors the standard ISO-14577 [5] recommends to use laser interferometry. However, this is costly, requires expertise in laser technology and there may be difficulties to place interferometers suitably. As an alternative, imprints on reference samples can be used to calibrate the depth sensor by another suitable tool.

Atomic force microscopy (AFM) is a popular tool for length measurements at the nanoscale. It works usually in ranges up to approx. $10 \mu \mathrm{m}$ in the vertical direction, thus covering a range of imprints on various samples. It operates in air and does not require any specific sample preparation. Traceability can be achieved with suitable height standards.

In this contribution we study the feasibility of the calibration of depth sensors in nanoindenters using AFM measurements of imprints on samples. Results from calibrations of different instruments are presented and analyzed. Several sources of uncertainties must be analyzed. Uncertainties from the determination of the residual depth from nanoindentation unloading curves as well as from AFM images arise. Contamination also acts as a source of uncertainty. These uncertainties are presented and commented. Some estimates are given, although they depend on the instrument and sample. We also comment on the choice of the sample.

\section{EXPERIMENTAL}

Nanoindentations were performed on TI 950 nanoindenters from Hysitron located at and one Ultra Nano Hardness Tester from Anton Paar (formerly CSM). The AFM measurements were done using Dimension Icon microscopes from Bruker. Copper and fused silica reference samples were used. Some of the measurements were done in clean room facilities so as to reduce contamination.

\subsection{Nanoindentation}

Two TI 950 nanoindenters and one UNHT tester were used to create the imprints at different loads. Loads ranged from 1 to $10 \mathrm{mN}$, each load was used 5-9 times. Linear loading was used with both loading and unloading times $2 \mathrm{~s}$ (TI 950) or $30 \mathrm{~s}$ (UNHT). Berkovich tips were used in all cases. The residual depth was determined as the depth value for zero load. This corresponds to the point of last contact between the tip and the sample.

\subsection{AFM}

Two Dimension icon instruments were used. Measurements were performed in tapping mode. The data were corrected if necessary, e.g. levelling or row alignment. The depth of the imprint was determined as the distance between the minimum of the imprint and the mean plane surrounding it. A step height standard was used for the calibration of the z-sensor.

\section{EXPERIMENTAL}

A typical AFM measurement of an imprint on fused silica and copper is shown in Figure 1. The plane far away from the indent which acts as the reference plane was set to zero. Note also, that the roughness is higher for the copper sample. 


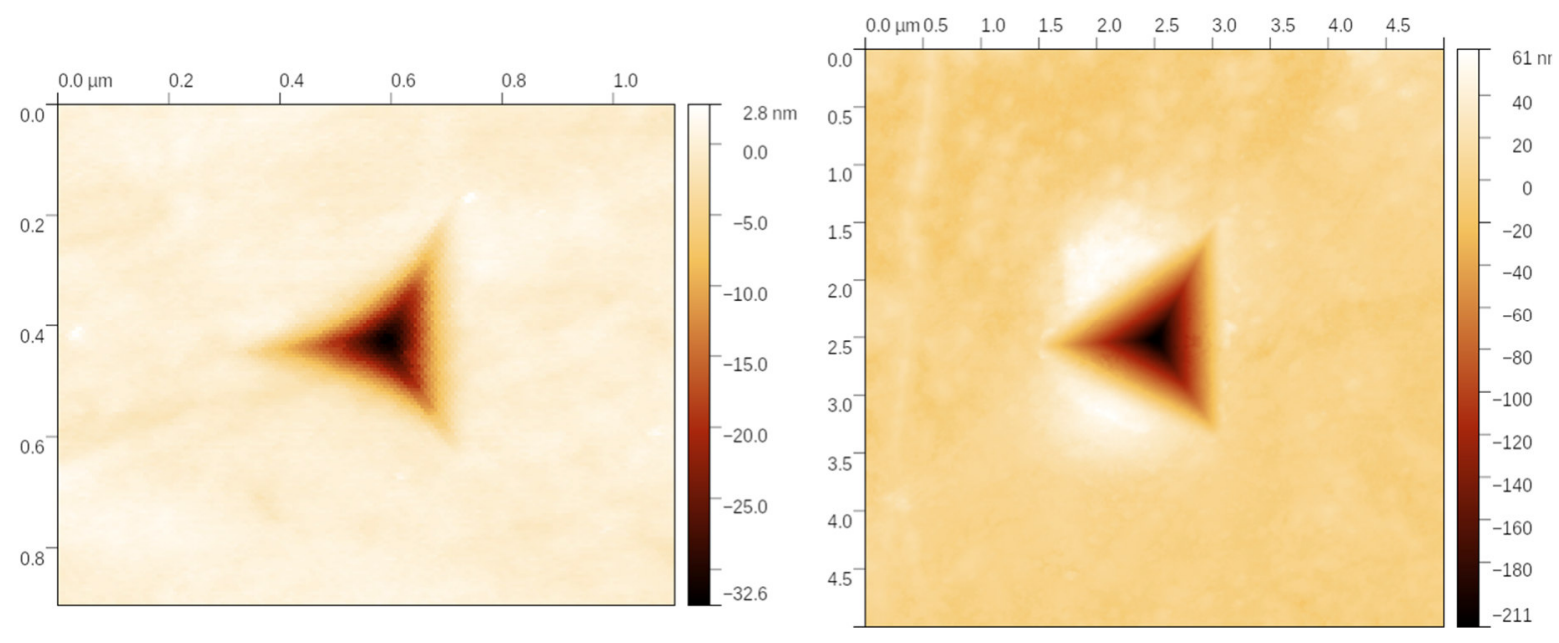

Figure 2 An AFM image of an imprint created at a load of $2 \mathrm{mN}$ on fused silica (left) and copper (right)

A typical loading-unloading curve on fused silica is shown in Figure 2. The inset shows a magnification of the regions marked in blue, where the tip loses contact with the sample. The point of last contact is shown in the inset in red. Note, the behavior of the load at the point of last contact. This is a feature of the feedback loop. The speed of the unloading and the sampling rate determine the number of data points available and thus also the step in depth. Since this directly determines the uncertainty, care should be taken to choose slow unloading with high data sampling. The last contact point was chosen either manually or using the intercept of a fitted line with the zero axis.

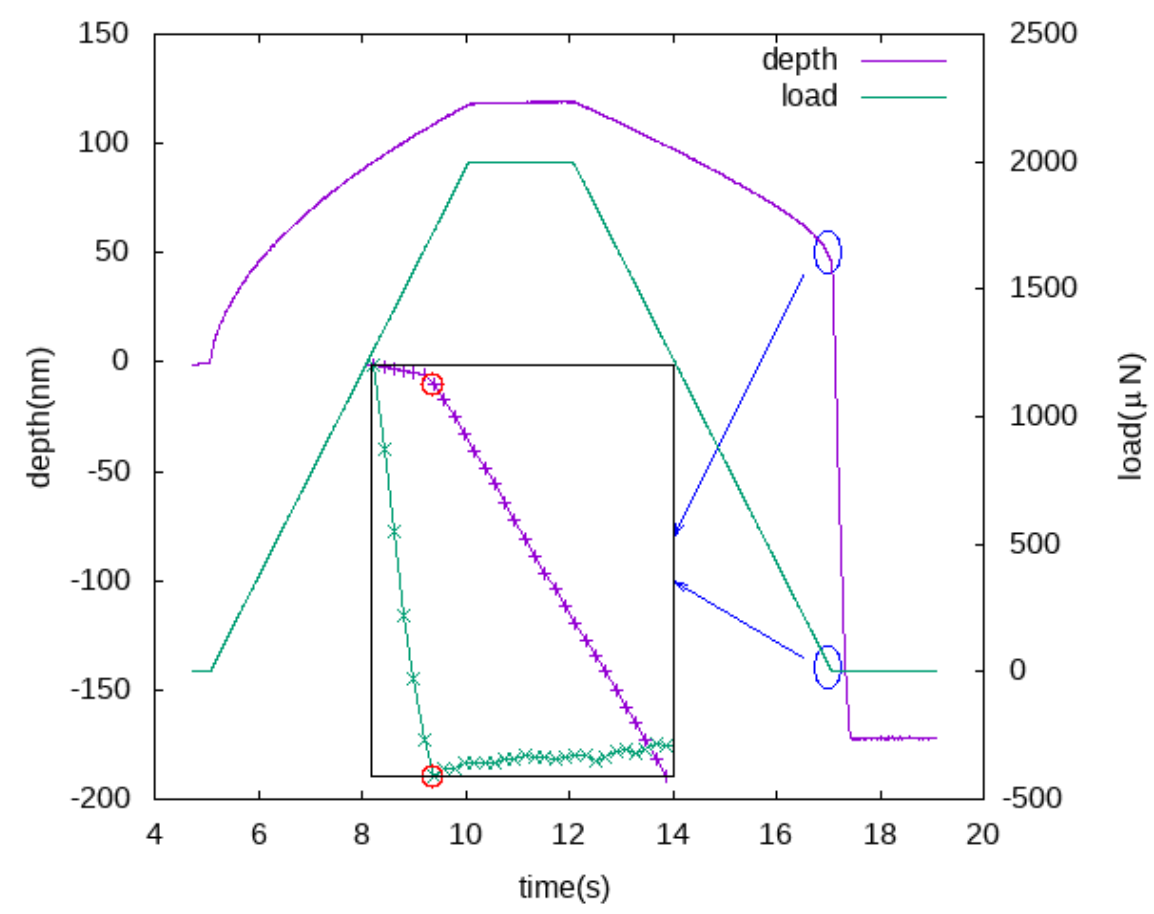

Figure 2 A typical loading/unloading curve on fused silica. The inset shows a magnification of the regions marked in blue, where the tip loses contact with the sample. The point of last contact is shown in the inset in red.

The depth data determined by AFM and nanoindentation for two instruments are combined in Figure 3. The data can be used to find e.g. a linear calibration relation. 

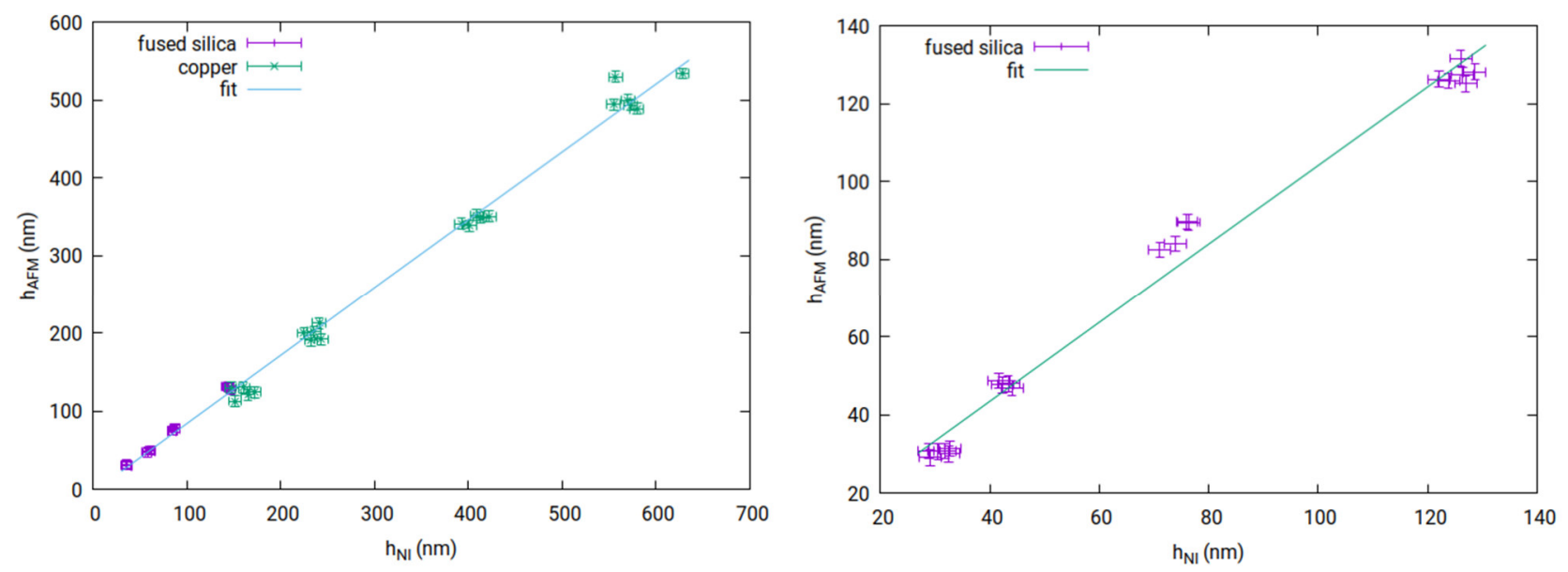

Figure 3 Depth values determined from nanoindenter and AFM for two different instruments including a linear fit. The relative uncertainty of the slope is $4 \%$ (left) and $3 \%$ (right).

\subsection{Uncertainties in the Nanoindentation method}

In this contribution, when determining the residual depth, we take into account three sources of uncertainty: uncertainty related to the determination of first contact, uncertainty related to the determination of last contact and the elastic deformation due to the uncertainty in load. The determination of contact depends crucially on the sampling rate and to a lower degree also on the approach or unloading rate, i.e. the speed of the tip with which it is approaching the sample or is being retracted. The contact criterion, such as the definition of the contact force plays a minor role. We found that the uncertainties of first contact were negligible for the TI 950 instrument, being around $0.2 \mathrm{~nm}$. For the UNHT, on the other hand, the choice is much more critical. It could be kept below $1 \mathrm{~nm}$ with suitable settings but easily exceed $2 \mathrm{~nm}$ for the UNHT for non-optimal settings. We found uncertainties of last contact around $1 \mathrm{~nm}$ but also significantly higher values up to $5 \mathrm{~nm}$ when the settings were not chosen well. A small elastic deformation remains up to the final separation of tip and sample thus leading to an overestimate of the residual depth. Since the unloading is equivalent to a purely elastic interaction with an effective indenter tip [6] we estimated the residual deformation based on the formula (1) from Hertz contact theory [7]

$h=\left(\frac{3}{4} \frac{F}{E \sqrt{R}}\right)^{2 / 3}$

where $F$ is the residual load, $E$ is the modulus of reduced contact and $R$ is the tip radius. As shown in [8], the effective tip shape tends to have a radius at least as high as the original tip. This allows us to estimate upper bonds for the residual deformation. Assuming a residual load of $2 \mu \mathrm{N}$ (TI 950) or $10 \mu \mathrm{N}$ (UNHT) and a tip radius between 50 and $150 \mathrm{~nm}$, we arrive at a deformation between $1.5-2.0 \mathrm{~nm}$ for fused silica and $2.8-4.0 \mathrm{~nm}$ for copper. The literature values for the moduli of reduced contact are $70 \mathrm{GPa}$ for fused silica and $130 \mathrm{GPa}$ for copper.

\subsection{Uncertainties in the AFM method}

Several effects must be taken into account when determining the overall uncertainty of the depth of the imprint. The roughness of the sample contributes to the uncertainty of the flat plane outside of the imprint. Contamination and the resolution affect the determined minimum depth value. The uncertainty of the calibration of the z-sensor contributes to the overall depth value. The roughness was below $1 \mathrm{~nm}$ for fused silica and 5-8 $\mathrm{nm}$ for copper. Bigger particles of contamination on the flat part can be automatically or manually detected and removed from the evaluation of the reference plane. As the AFM gives us values on a grid, the minimum itself probably won't be measured at. This means, we need a sufficient resolution to ensure that the 
error by substituting the real minimum by a close value will be small enough. Assuming a spherical shape of the imprint around the minimum with a radius of 50-150 nm the pixel size should not be larger than approx. 15 $\mathrm{nm}$ in order to keep the uncertainty below $2 \mathrm{~nm}$. The dependence on the resolution was verified by measuring one indent repeatedly with different resolution. We found differences in the range of 1-2 nm depending on the size of the imprint. If the contamination is close to the minimum in the imprint itself, the depth of the imprint can no longer be determined exactly. Fortunately, even in this case the induced error may not be too dramatic thanks to the bluntness of the indent. This is illustrated in Figure 4, where the real shape of the indent was estimated and the difference found to be approx. $1 \mathrm{~nm}$. The repeatability was tested by remeasuring a set of imprints. Differences of over $3 \mathrm{~nm}$ were found, thus indicating the necessity of proper cleaning and data acquisition. The uncertainty of the z-sensor calibration constant was mostly around $1 \%$.

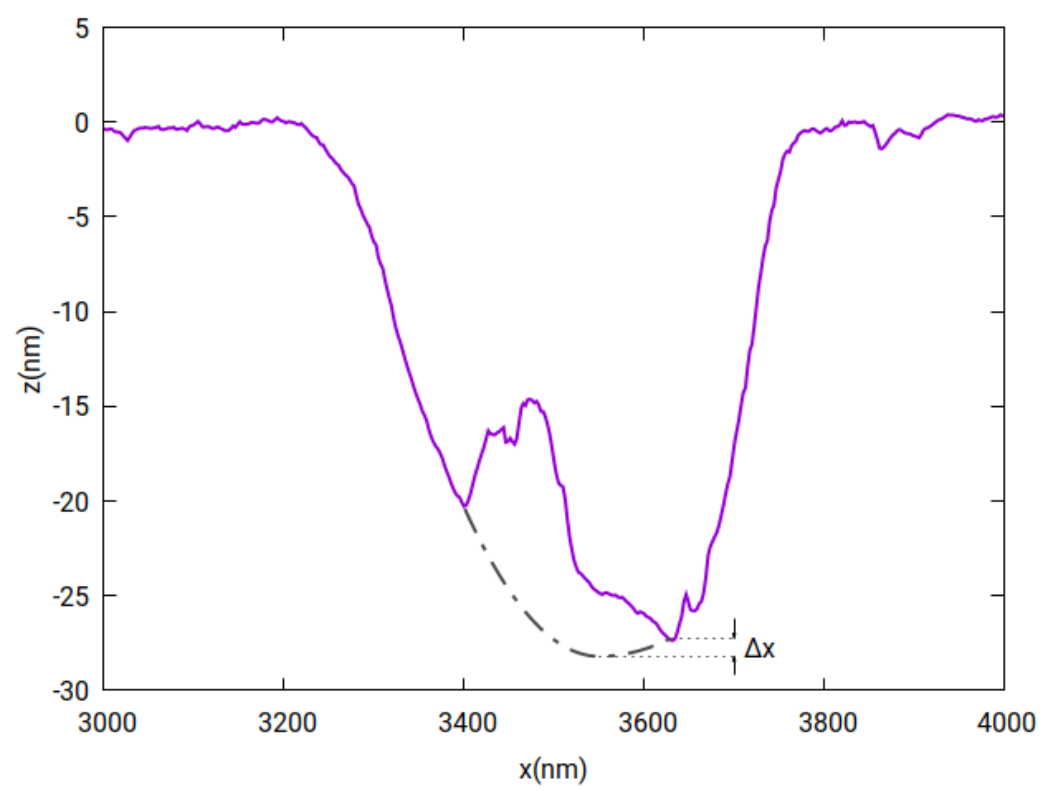

Figure 4 Example of a profile across a contaminated imprint with estimated reconstructed shape. The difference in this case is $1 \mathrm{~nm}$.

\section{DISCUSSION}

In this work we used fused silica and copper samples, in previous work we used also steel reference samples. Several aspects should be considered when choosing a sample. The hardness of the sample should be such that the created imprint can be measured by the AFM with a reasonable uncertainty. If the sample is very hard, the imprints will be very shallow. Since the dominant sources of the AFM uncertainty do not depend on the depth itself, the relative uncertainty will be very high. On the other hand, if the sample is very soft, the imprints will be too deep making them impossible to measure by AFM. The elasticity should be low as it acts as a source of uncertainty for the determination of the residual depth from the nanoindenter's unloading curve. The roughness of the sample makes the determination of the reference plane in the AFM measurement more difficult. Several samples can be combined to cover a larger depth range.

Contamination is a significant problem. If the nanoindentation tip happens to make contact with the contamination first, this will lead to an erroneous determination of the zero contact. This can be reduced by cleaning the sample and carefully inspecting and choosing a suitable area. The change in the surface energy after creating an imprint attracts the contamination particles to the imprints. The contamination on the flat surface also adds uncertainty to the determination of the flat plane, although this can be reduced by suitable filtering procedures. As mentioned above, contamination of the imprint itself can alter the apparent position of 
the minimum. Contamination also contributes to problems with the feedback loop in the AFM or with the AFM tip. In order to reduce contamination, the sample should be moved from the nanoindenter to the AFM as fast as possible. Measurements in clean room facilities are preferable.

Most sources of uncertainty do not depend on the depth value. This means that their influence will be large for shallow depths. Care must be taken to find suitable measurement settings both for AFM and for the nanoindentation as the uncertainties for standard settings may be too high.

The AFM measurements make this method quite time consuming. Imprints must be measured with high precision and at high resolution. Sufficient numbers of imprints should be made for each load value so that the data can be evaluated statistically.

\section{CONCLUSION}

The depth sensor of a nanoindentation instrument can be calibrated using AFM measurements of imprints on a suitable sample. Unlike direct calibration of the sensors which requires special equipment and expertise this method requires only an AFM which are often available in nanotechnology facilities. No special requirements are imposed on the sample. This makes the method accessible to wide range of scientists.

\section{ACKNOWLEDGEMENTS}

This work was supported by the Czech Science Foundation project GACR 19-15240S "Multifunctional Nanocomposite Polymer Thin Films with Controlled Surface and Mechanical Properties Deposited in RF Dusty Plasma“. Part of the work was carried out with the support of CEITEC Nano Research Infrastructure (ID LM2015041, MEYS CR, 2016-2019), CEITEC Brno University of Technology.

\section{REFERENCES}

[1] OLIVER, W. C. and PHARR, G. M. An improved technique for determining hardness and elastic modulus using load and displacement sensing indentation experiments. J. Mater. Res. 1992. vol. 7, pp. 1564-1583.

[2] ISO/IEC. Uncertainty of measurement - Part 3: Guide to the expression of uncertainty in measurement (GUM:1995). ISO/IEC Guide 98-3:2008. Geneva: International Organization for Standardization, 2008. 120 p.

[3] VANLANDINGHAM, M. R. Review of Instrumented Indentation. J. Res. Natl. Inst. Stand. Technol. 2003. vol. 108(4), pp. 249--265.

[4] CAMPBELLOVÁ, A., VALTR, M., ZU゚DA, J. and KLAPETEK, P. Traceable measurements of small forces and and local mechanical properties. Meas. Sci. Technol. 2011, vol. 22, pp. 094007-094013.

[5] Metallic materials - Instrumented indentation test for hardness and materials parameters, ISO 14577.

[6] PHARR, G. M. and BOLSHAKOV, A. Understanding nanoindentation unloading curves. J. Mater. Res. 2002, vol. 17. pp. 2260-2671.

[7] JOHNSON, K.L. Contact mechanics. Cambridge: Cambridge University Press, 1985. Cambridge.

[8] MERLE, B., MAIER, V., GOKEN, M. and DURST, K., Experimental determination of the effective indenter shape and $\varepsilon$-factor for nanoindentation by continuously measuring the unloading stiffness. J. Mater. Res. 2012, vol. 27. no. 1, pp. 214-221. 